\title{
La formación ciudadana en la escuela: Problemas y desafíos
}

\author{
Civics Education at Schools: Problems and Challenges
}

\author{
Carlos Muñoz Labraña \\ Universidad de Concepción \\ Concepción, Chile \\ carlosem@udec.cl \\ Bastián Torres Durán ${ }^{2}$ \\ Universidad de Concepción \\ Concepción, Chile \\ bastiantorres@udec.cl
}

Recibido 9 de enero de 2014 • Corregido 20 de marzo de 2014 • Aceptado 2 de abril de 2014

Resumen. El artículo informa los resultados de una investigación financiada por el Ministerio de Educación de Chile (FONIDE N.o 310894) y por el Centro de Investigación Avanzada en Educación, CIAE (FONDAP N. 11-2009). El estudio tuvo como objetivo investigar la percepción de los estudiantes respecto de la formación ciudadana que, de acuerdo con el currículo escolar, deben recibir en la escuela; esto, con el propósito de detectar los problemas y desafíos que - de estas percepciones-se desprenden para el profesorado de historia y geografía en la educación general básica. La metodología utilizada fue de carácter cualitativa y fenomenológica. La muestra fue de tipo intencional y estuvo constituida por estudiantes de ambos sexos de octavo año, pertenecientes a doce escuelas de educación básica de distinta dependencia administrativa, pertenecientes a la comuna de Concepción-Chile. El instrumento para recoger la información fue una entrevista en profundidad del tipo semiestructurada y para su análisis se empleó el programa Atlas.ti. Los resultados obtenidos son muy poco auspiciosos, puesto que, a pesar de los recientes esfuerzos ministeriales que complementan las innovaciones efectuadas en la década pasada en materia de formación ciudadana, el estudiantado no posee una adecuada concepción de lo que es la formación ciudadana ni de lo que se pretende con esta, tampoco la perciben como un espacio que los ayude en esta formación.

Palabras claves. Educación, ciudadanía, formación ciudadana.

1 Investigador principal del Proyecto FONIDE 310894 y FONDAP N.o 11-2008. Director Programa Magíster en Educación Universidad de Concepción. Docente Departamento de Ciencias de la Educación de la Facultad de Educación de la Universidad de Concepción. Chile. Investigador asociado del Centro de Investigación Avanzada en Educación CIAE, Chile. Miembro del Grupo Interdisciplinario de Investigación en Derechos Humanos y Democracia (GIIDHD) UDEC 12.E4.03. El artículo fue financiado por el proyecto UCO 1203 "Profesores UDEC: Protagonistas del cambio en la sociedad del conocimiento".

2 Licenciado en Educación con mención en Historia y Geografía. Ayudante de la cátedra de Principios de Educación I y II de la Facultad de Educación de la Universidad de Concepción. Ayudante en el Grupo Interdisciplinario de Investigación en Democracia y Derechos Humanos (GIIDHD) UDEC 12.E4.03. Tutor en el Centro de Ayuda al Desarrollo del Estudiante de la Facultad de Educación (CADE FE). 
doi: http://dx.doi.org/10.15359/ree.18-2.12

URL: http://www.una.ac.cr/educare

CORREO: educare@una.cr

Abstract. This article reports the results of a research project financed by the Chilean Ministry of Education (FONIDE No. 310894) and the Center for Advanced Research in Education (AEIC) (FONDAP No. 11-2009). The aim of this research project was to investigate the students' perception regarding civics education, which, according to school curricula, should be taught in school, in order to detect the problems and challenges resulting from those perceptions, which will be faced by history and geography teachers in schools. The methodology used was qualitative and phenomenological. Samples were selected using an intentional sampling method and included male and female eighth graders from twelve schools from different administrative units in the Concepción district in Chile. Information was obtained using a thorough semi structured interview and analyzed using the Atlas.ti Software. Results were not very promising since, despite the Ministry's recent efforts, which included changes made during the last decade to civics education, students do not have an adequate notion of what civics education is or what it is for. Neither do they consider these classes a space that helps them in this respect.

Keywords. Education, citizenship, civics education.

\section{Introducción}

Si bien no cabe duda de que la escuela no es la única institución que contribuye a la formación de la ciudadanía en una sociedad y que, por tanto, por sí sola no puede llegar muy lejos, su papel en estos procesos formativos es crucial. La razón es que la escuela tiene como una de sus metas desarrollar, en la niñez y la juventud, aquellas habilidades y actitudes que les permiten entender y aprender a vivir en sociedad, promoviendo el respeto y la defensa de los derechos humanos, así como la valoración y práctica del ideal democrático al que debería aspirar toda sociedad (Bolívar, 2007).

La escuela plantea, como intención propia, constituir los espacios y organizar los tiempos en que tiene lugar la primera experiencia común a toda la sociedad (Cerda, Loreto, Magendzo, Santa y Varas, 2004). De tal manera que "puede contribuir, decisivamente o no, a construir las capacidades intelectuales y morales que son fundamento de la capacidad de acción... ciudadana de la sociedad. Es decir, de reflexión y acción sobre sí misma: sus problemas más apremiantes, sus fines, y los significados que la cohesionan (Cox, Jaramillo y Reimers, 2005, p. 11).

En la escuela se experimenta la alteridad y la otredad y se adquieren, al mismo tiempo, algunas de las virtudes imprescindibles para la vida compartida (MINEDUC, 2004c). Tal como plantean Andrade y Miranda (2000, citando a Martinic, 1995):

Aquí, se constituyen los conocimientos compartidos y elaborados socialmente para aprehender lo real y dar sentido a las prácticas cotidianas de los individuos; permite clasificar, distinguir, ordenar, evaluar y jerarquizar un estado de la realidad o de una dimensión de la vida cotidiana, ... [haciendo] lógico y coherente el mundo, organizando las explicaciones sobre los hechos y las relaciones causales que existen entre ellos. (Andrade y Miranda, 2000, p. 3, citando a Martinic, 1995) 


\section{Marco teórico}

Desde el punto de vista curricular, Chile ha realizado un gran esfuerzo con el propósito de promover la formación ciudadana en la niñez y la juventud, pretendiendo superar las graves omisiones heredadas del gobierno militar, cuyo currículo fue deficitario especialmente respecto de la consideración de algunos conceptos claves asociados a la democracia y a los derechos humanos, así como a otros relacionados con el aprendizaje de vida en común (Muñoz, Vásquez y Reyes, 2010). Este vacío curricular quedó en evidencia en una medición internacional de educación cívica efectuada en 1999, en donde un tercio de las preguntas de la prueba realizada por los estudiantes chilenos se referían a materias no contempladas en programa de estudio alguno (Muñoz, Ajagan, Sáez, Cea y Luengo, 2013).

Con posterioridad a la recuperación de la democracia en el país, se han realizado importantes innovaciones curriculares que modificaron la orientación del currículo escolar, los programas de estudio, la evaluación y la mediación pedagógica, con el objetivo de reforzar e incorporar una serie de contenidos relacionados con la democracia y los derechos humanos $y$, además, desarrollar algunas importantes habilidades y actitudes en el estudiantado, destinadas a favorecer y fortalecer aquellos principios y valores fundamentales asociados a estas temáticas (MINEDUC, 2004b).

La decisión del Ministerio de Educación (MINEDUC) de incorporar nuevas temáticas y vincular la formación ciudadana con problemas actuales de la sociedad de manera inclusiva, comprensiva y participativa es coherente con el enfoque maximalista descrito por la bibliografía especializada (Kerr, 2002). Y ha tenido como objetivo "abrir una brecha epistemológica en el sistema educativo orientado tradicionalmente a las estructuras curriculares basadas en lógicas disciplinares creadas en una tradición cultural de hace siglos" (MINEDUC, 2004b, p. 25).

Con el propósito de llevar al aula esta nueva forma de entender la formación ciudadana, se incorporan nuevos conocimientos, habilidades y actitudes en la asignatura de historia y geografía. La razón fue que en ella existían objetivos coherentes al marco de valores que debían inspirar a las personas ciudadanas en el Chile contemporáneo, buscando que el estudiantado valore el pluralismo y logre desarrollar una actitud positiva hacia la participación ciudadana (MINEDUC, 2004b), mediante la incorporación de conocimientos y habilidades asociadas, como el fomento del desarrollo del pensamiento crítico e independiente; la incorporación de metodologías orientadas a la discusión grupal; la promoción de resolución de problemas y el análisis de casos concretos, vinculados a su aquí y ahora; el énfasis en los procesos conscientes al abordar los contenidos mediante el análisis, la demostración y la argumentación, y la utilización de gran variedad de materiales de aprendizaje (mensajes de los medios de comunicación, discursos, graffitis, etc.).

Muñoz, Ajagan et al. (2013, p. 132, citando a Lundgren, 1997, p. 21), señalan que en el contexto del diseño curricular "los Programas de Estudio, los documentos oficiales de un Ministerio que regulan y norman la enseñanza por medio de un marco curricular nacional, pertenecen de acuerdo a la teoría curricular a una esfera de acción designada como la arena de la formulación" (Lundgren. 1997, p. 21 citado en Muñoz, Vásquez y Sánchez, 2013). 
doi: http://dx.doi.org/10.15359/ree.18-2.12

URL: http://www.una.ac.cr/educare

CORREO: educare@una.cr

Sin embargo, lo que sucede en el aula no es las más de las veces lo que manifiestan los programas de estudio y las directrices y normativas oficiales.

Entre el plano de la formulación -es decir, en el de los documentos oficiales- y lo que ocurre en el aula existe una serie de acciones e instrumentos de mediación que operan como verdaderos filtros, que, la mayoría de las veces, modifican la propuesta curricular original, entre los que destacan: el uso dado al lenguaje; la pertinencia del perfeccionamiento docente; la mayor o menor consideración de los libros de texto; la utilización de estrategias con énfasis en la enseñanza o el aprendizaje; el tipo de cogniciones distribuidas entre el estudiantado, etc. (DíazBarriga y Hernández, 2005). Este plano es conocido con el nombre de "arena de la transformación" (Lundgren, 1997), porque será en torno al aula donde el profesorado concretizará, en mayor o menor medida, las indicaciones oficiales. Es en esta última donde los estudiantes tienen mucho que decir (Muñoz, Agajan et al. 2013).

Muñoz, Vásquez y Sánchez (2013, p. 97) indican que:

La [recontextualización] del currículum significa que, si bien los Programas de Estudio pueden propiciar y proponer ciertas formas de enseñanza, promover el aprendizaje de determinados conocimientos declarativos, enfatizar el desarrollo detalesocuales destrezas, habilidades e incluso valoraciones, en la realidad del aula el profesorado --al interpretar y concretar con su práctica las indicaciones efectuadas en la arena de la formulación[bien] puede [incluso] usar formas de enseñanza reñidas con las orientaciones oficiales. Esto es generado porque el profesor posee una cierta libertad de acción para interpretar los Programas de Estudio y concretizarlos en el aula, fenómeno que es conocido con el nombre de "autonomía del profesor". (Apple, 2004 p. 28)

Investigaciones efectuadas por el Ministerio de Educación de Chile (MINEDUC, 2004c), con el propósito de hacer un seguimiento de la implementación curricular, concluyen que el marco curricular ofrece una sólida base de sustentación para hacer de la escuela un espacio de formación ciudadana. Sin embargo, en un Estudio Internacional de Educación Cívica, realizado por la International Association for the Evaluation of Educational Achievement, en el año 1999 y en el año 2010, se detectaron varios ámbitos consultados en donde los estudiantes no logran superar la media internacional (MINEDUC, 2004a).

Los antecedentes anteriores llevan a dos preguntas fundamentales: ¿Cuáles son las percepciones que hoy poseen los estudiantes acerca de la formación ciudadana -que de acuerdo con el currículo escolar- deben recibir en la escuela? ¿Es posible detectar algunos problemas y desafíos para el profesorado de historia, geografía y ciencias sociales en estas percepciones?

No cabe duda de que la formación ciudadana depende, quizás más que de ningún otro factor, de la preparación de su profesorado. El personal docente es el que, en última instancia, traduce el currículo declarado en currículo aplicado (Lundgren, 1997)."A pesar de la importancia de los docentes en la temática, recientes investigaciones demuestran que el espacio y el 
doi: http://dx.doi.org/10.15359/ree.18-2.12

URL: http://www.una.ac.cr/educare

CORREO: educare@una.cr

tiempo curricular que ocupan los conocimientos que responden a la racionalidad instrumental, sobrepasan con creces a los conocimientos que apuntan a una racionalidad axiológica y comunicativa tendiente a formar sujetos de derechos, como los que se promueven desde una educación que tiene como objetivos la formación ciudadana" (Magendzo, 2004, p. 36).

Por otro lado, "el propio Ministerio de Educación, ha constatado que el componente de formación ciudadana de la Reforma Curricular es uno de los menos comprendidos por los docentes existiendo numerosas tensiones que han sido ratificadas por otros estudios posteriores" (Egaña, 2003, p.12). Además, en una reciente investigación realizada en el país, se concluyó que al finalizar el segundo ciclo básico, los grupos estudiantiles asocian el rol del Estado a cuestiones más sociales que políticas y que todos, sin excepción, manifiestan una gran desconfianza en los partidos políticos (Muñoz, Victoriano, Sánchez y Wilhelm, 2010).

\section{Metodología}

La metodología utilizada para llegar a identificar las percepciones de los estudiantes ha sido cualitativa y fenomenológica (Stake, 2010). Se parte del supuesto básico de que el mundo social es un mundo construido con significados y símbolos, de tal manera que lo que se pretendió en la investigación fue buscar esa construcción y sus significados.

La muestra ha sido de tipo intencional y ha estado constituida por estudiantes de doce escuelas de educación básica de distinta dependencia administrativa (4 particulares, 4 subvencionadas y 4 municipales). Todas ellas pertenecen a la comuna de Concepción-Chile y atienden niños de ambos sexos con edades fluctuantes entre los 14 y los 15 años.

En cada establecimiento se trabajó en un octavo año básico elegido al azar y en las asignaturas de historia, geografía y ciencias sociales, que poseen 3 horas pedagógicas de 40 minutos a la semana en cada curso.

El instrumento para recoger la información fue la entrevista en profundidad del tipo semiestructurada, básicamente, porque posee la flexibilidad en su guión para ir agregando nuevos temas o problemas a lo largo de la entrevista, lo que favorece la profundización de las temáticas. En total se realizaron 24 entrevistas (12 mujeres y 12 hombres; uno y una por cada establecimiento). Los estudiantes entrevistados fueron elegidos al azar, de entre aquellos que asistieron al día en que se efectuó la visita, previamente acordada con las autoridades del establecimiento.

Las entrevistas se efectuaron en una oficina facilitada por las autoridades de cada uno de los establecimientos de la muestra. Fueron grabadas en audio y luego transcritas en Microsoft Word.

Efectuado el trabajo anterior, se procedió al análisis del material disponible, a través del "análisis de contenido por categorías", con una orientación hermenéutica o interpretativa. Para un óptimo análisis de contenido, se siguieron los pasos aconsejados 
doi: http://dx.doi.org/10.15359/ree.18-2.12

URL: http://www.una.ac.cr/educare

CORREO: educare@una.cr

por Bardín (1996). Estos inician con un "preanálisis de la información", correspondiente a "la fase de organización propiamente dicha y corresponde al período de intuiciones" (p. 71); continúan con una etapa de "codificación de la información", en tanto "proceso por el que los datos brutos son transformados sistemáticamente y agregados en unidades que permiten una descripción precisa de las características pertinentes del contenido" (p. 78); y finalizan con una "categorización de la información", consistente en clasificar los "elementos constitutivos de un conjunto por diferenciación, tras la agrupación por ... (analogía), a partir de criterios previamente definidos (p. 90). Para facilitar esta importante tarea se utilizó el programa Atlas.ti.

\section{Resultados}

Considerando las preguntas de investigación y el análisis que emerge del material empírico procesado a través de Atlas.ti, los resultados se han organizado en dos grandes grupos. El primero reúne aquellas percepciones del estudiantado vinculadas a la formación ciudadana que reciben en la escuela. El segundo da cuenta de los principales problemas y desafíos que se desprenden del discurso de los estudiantes, para el profesorado de la especialidad.

Dos grandes categorías emergen en relación con la percepción que poseen los estudiantes sobre la formación ciudadana que reciben en la escuela, las que para mayor claridad han sido resumidas en la figura 1.

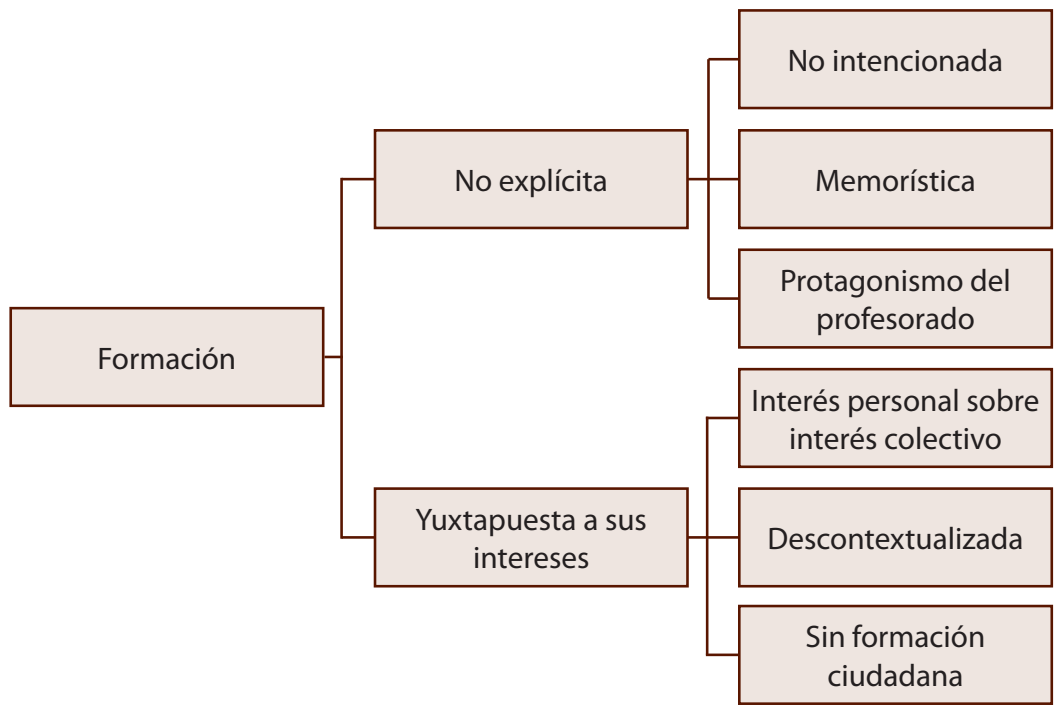

Figura 1. Categorías asociadas a la formación ciudadana recibida en la escuela. Elaboración propia a partir de los resultados arrojados por el programa Atlas ti. 


\section{Una formación no explícita}

A pesar de que la formación ciudadana está claramente relevada en el currículo de formación del nivel, el estudiantado percibe que esta no ha sido recibida de manera explícita ni intencionada en las actividades que cotidianamente realizan en la escuela, puesto que, en general, reconocen que en esta se han dedicado, fundamentalmente, a escribir largas listas de contenidos que, en su mayoría ,deben memorizar y que, a lo más, culminan en el desarrollo de un cuestionario, cuyas preguntas en su mayoría son realizadas por el profesorado.

No obstante lo anterior, los estudiantes reconocen que, dentro de los contenidos entregados, existen algunos conocimientos y ciertas habilidades asociadas como: la capacidad de ser tolerantes, de respetar las ideas ajenas y de trabajar de manera colaborativa. Sin embargo, no logran relacionarlas ni contextualizarlas desde un contexto social más amplio. El estudiantado las valora porque las considera útiles para su formación como individuos, para su crecimiento y bienestar personal, y no porque ellas pudieran contribuir a su formación como ciudadanos y ciudadanas. El eje de la valoración es el individuo y no el aporte que puede realizar ese individuo al resto de la sociedad.

\section{Una formación que se yuxtapone con sus intereses}

La falta de contextualización de las actividades efectuadas en torno a un eje que favorezca la formación ciudadana del estudiantado y, por tanto, su inserción plena en la sociedad, hace que el alumnado yuxtaponga sus intereses con los de la sociedad. De esta manera se levantan dos bloques que no dialogan ni se interrelacionan, porque por una parte están sus intereses $y$, por otro muy distinto, los intereses de la sociedad.

Los estudiantes no realizan el puente, el enlace que les permita comprenderse como miembros de una sociedad, de allí que hablen: de la sociedad, más que de nuestra sociedad; de lo importante que es concretar anhelos personales, más que societarios; de proteger sus intereses personales, más que los del grupo; de la trascendencia de cumplir sus legítimas aspiraciones, más que los de la sociedad; de la importancia de lograr un bienestar personal, más que del grupo; de adquirir y proteger sus bienes materiales, más que los de la sociedad; de cuidar el mobiliario familiar, más que el mobiliario público que utilizan a cada momento, y de la importancia del capital financiero, más que del capital social. En fin, hablan desde sí y para sí.

Este lenguaje individualizado hace que ciertas virtudes cívicas asociadas por el estudiantado al concepto de formación ciudadana -como la responsabilidad, por ejemplo- se asuma frente a otro u otros, pero no con otros. Cuestión que resulta coherente con lo planteado por algunos autores, que han hecho ver el excesivo individualismo con que los sujetos en nuestra sociedad encarnan los problemas cotidianos (Bauman, 2005). Al parecer los estudiantes esperan hallar soluciones individuales para problemas construidos socialmente (Beck, 1993). 
doi: http://dx.doi.org/10.15359/ree.18-2.12

URL: http://www.una.ac.cr/educare

CORREO: educare@una.cr

Las categorías asociadas a la formación -que han sido consignados en la figura 2- nos permiten identificar los siguientes desafíos para el profesorado.

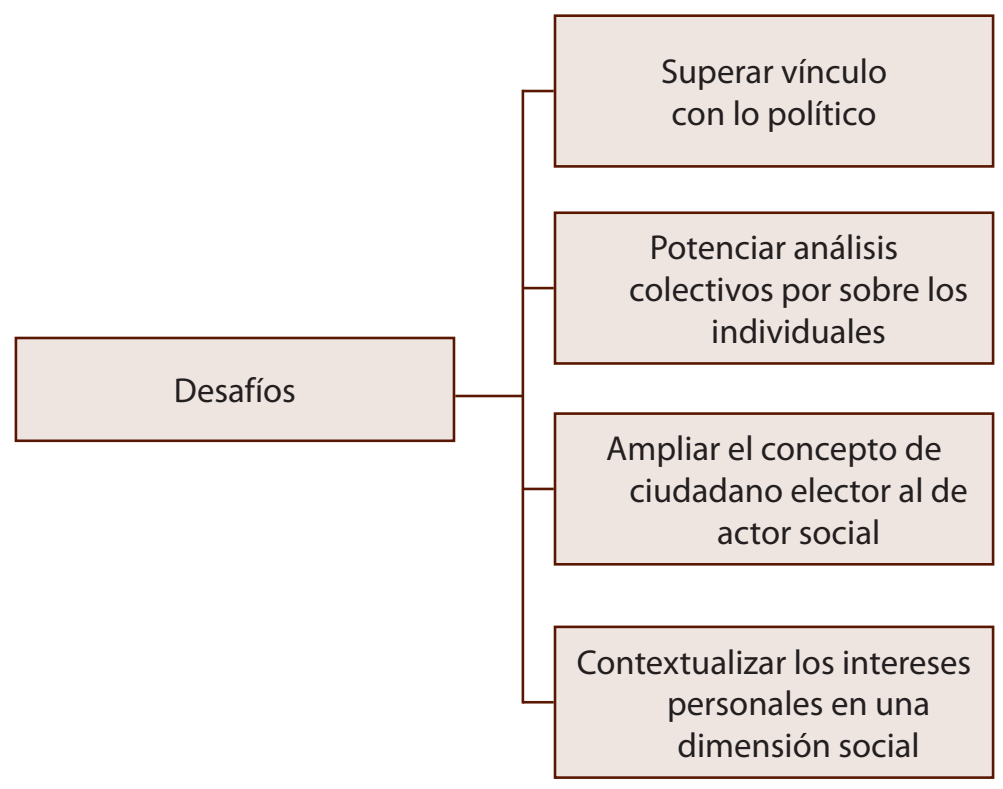

Figura 2: Categorías asociadas a los desafíos para el profesorado. Elaboración propia a partir de los resultados arrojados por el programa Atlas ti.

\section{Superar el vínculo con lo político}

La única aproximación vinculada a la formación ciudadana que el estudiantado logra balbucear está asociada a la posesión de ciertos deberes, siempre mirados desde un punto de vista estrictamente político y legal, en donde la posibilidad de participar en elecciones ocupa un lugar preponderante y central. Cuestión que es propia de los enfoques minimalistas descritos por la bibliografía (Kerr, 2002).

A veces algunos estudiantes, los menos, homologan la expresión de formación ciudadana a la de educación cívica, que alguna vez han escuchado en su realidad escolar ${ }^{3}$. Sin embargo, ven esta educación cívica de manera eminentemente prescriptiva y factual. No la relacionan con el desarrollo de ciertas habilidades ni menos actitudes vinculadas al aprender a vivir en sociedad. Solo la asocian a un área dentro de las ciencias sociales que estudia las obligaciones prescritas en un cuerpo legal como la Constitución Política, la cual tiene, como fuente de inspiración,

3 La educación cívica fue una asignatura impartida durante el Gobierno Militar y tanto los profesores como los padres de los estudiantes estudiaron esta asignatura en sus años de escolaridad obligatoria. 
doi: http://dx.doi.org/10.15359/ree.18-2.12

URL: http://www.una.ac.cr/educare

CORREO: educare@una.cr

ciertos conceptos e ideas que alguna vez han tenido la oportunidad de leer o escuchar en alguna parte, pero sin una genuina apropiación de estas, desde el punto de vista conceptual. De hecho, hacen el esfuerzo de conceptualizar, pero no poseen herramientas que les permitan aplicarlos a su cotidianeidad, ni menos advertir su importancia y relación para la vida de la sociedad a la que sin duda pertenecen ${ }^{4}$.

\section{Potenciar los análisis colectivos por sobre los individuales}

En la asociación [conceptual] efectuada por el estudiantado entre formación ciudadana y educación cívica, destaca el carácter utilitario que le dan a esta educación cívica, así como su asociación con lo que algunos autores denominan virtudes cívicas Cortina (1997), que sin duda están asociadas a ciertos valores morales, como la libertad, el respeto, la tolerancia y la justicia. Sin embargo, estas virtudes cívicas, siempre son vistas por el estudiantado desde un punto de vista individual, haciendo dos grandes abstracciones. La primera, está relacionada con la desconsideración del carácter dialógico que posee el propio ejercicio cívico y la segunda, con una ausencia del otro y de los otros, [a los cuales evidentemente el estudiantado pertenece]. De esta manera, se produce una suerte de ocultamiento de las responsabilidades conjuntas y compartidas, de la corresponsabilidad y de la participación en lo público. Lo que coincide con resultados de otras investigaciones [existentes en el área] (Valderrama, 2007). (Muñoz, Victoriano, et al (2010, p. 4)

\section{Ampliar el concepto de ciudadano elector al de actor social}

El estudiantado homologa el concepto de ciudadano o ciudadana al de elector o electora y a nada más. Ven a este ciudadano-elector con una óptica eminentemente política, legal y electoral, sin ir más allá. Construyen un imaginario integrado por una parte por este ciudadanoelector y por otra por el acto o evento electoral. En esta relación tiene gran importancia la acción de votar y, por supuesto, el voto asociado a esta acción, el que entienden como la expresión de una persona particular, que desea hacer ver su apoyo a tal o cual persona que posee la calidad de candidato o candidata.

Desde esta perspectiva, el voto constituye un puente entre la ciudadanía y la sociedad, mas no de su sociedad. Un punto de confluencia entre las opiniones individuales de los ciudadanos-electores y un ideario etéreo de sociedad, de la que no se sienten partícipes. A tal punto que cuando se refieren a ella, lo hacen siempre como algo paradójicamente ajeno a su cotidianeidad y vivencia personal.

$4 \quad$ Es importante recalcar la expresión a la que pertenecen, por cuanto una de las pretensiones de esta comunicación es advertir el lenguaje enajenador utilizado por los estudiantes a la hora de tener que pronunciarse sobre su aprendizaje ciudadano en el sistema escolar. 
doi: http://dx.doi.org/10.15359/ree.18-2.12

URL: http://www.una.ac.cr/educare

CORREO: educare@una.cr

Al asociar el voto a una opinión individual, los estudiantes establecen una relación de uno a uno. Lo anterior hace que en una contienda electoral triunfa quien logra tener más unos en torno a su persona y no aquella persona candidata que logra concitar cierta adhesión en torno a un proyecto de sociedad, o amalgama ciertas voluntades, o en torno de la cual se obtienen ciertos consensos acerca de un proyecto o ideario de sociedad.

La fuente de inspiración de este veredicto es eminentemente personal y se encuentra en algunas ideas poco estructuradas de lo que entienden debería ser lo mejor para la sociedad; pero en las cuales no se ven ni se sienten integrados, ni menos se logran o desean proyectar, más allá de sus naturales, legítimas y específicas aspiraciones personales, cuya procedencia proviene, en su mayoría, de exigencias del mundo familiar más cercano y no de una construcción amalgamada dentro de la institución escolar.

\section{Contextualizar los intereses personales en una dimensión social}

Las opiniones individuales realizadas por el ciudadano-elector, tal como se ha descubierto en otras investigaciones (Muñoz, Victoriano et al., 2010), provoca:

Una disyunción de intereses, que hace que el estudiantado vea la participación a través del voto o incluso de cualquier otra forma de participación dentro de la sociedad, como una relación disyuntiva y contrapuesta a sus proyectos personales, que los lleva a plantear que si se preocupan de la sociedad, podrían lesionarlos. De allí su reserva mayoritaria de no comprometerse a participar. No es que no reconozcan los desafíos que enfrenta la sociedad en nuestros días, tampoco que efectúen un diagnóstico muy equivocado de ella, sino que ven este desafío como una contraposición de intereses, que no logran armonizar. Hacen el diagnóstico pero se quedan en él. No se sienten con herramientas que les permitan proyectar un ideario que les ayude a situarse dentro de la sociedad, a la que pertenecen. Se sienten personas, pero de alguna manera se sienten 'alguien que aún no es (Valderrama, 2007), un sujeto inacabado que está en una etapa de construcción', que una vez cumplida cierta edad, podrá o no participar. (p. 6)

Ciertamente que, frente a esta disyunción, la opción del estudiantado es una sola: Preocuparse por sus proyectos personales, dejando para otros o para más adelante la opción de pensar en la sociedad que quieren vivir superando el paradigma personal. Pensar en su sociedad se convierte, entonces, en una opción de futuro, de un futuro que eventualmente podría no llegar.

Este último hallazgo resulta muy interesante de consignar, porque en el análisis del discurso del estudiantado es notorio el alto nivel de individualidad que alcanzan luego de ocho años de escolarización formal y de cómo es que al finalizar un ciclo de formación, se aleja la posibilidad de pensar en una vida en sociedad. 


\section{Conclusiones}

Los hallazgos de la investigación confirman la distancia que existe, en materia de formación ciudadana, entre el currículo formulado y el ejecutado. Esto, porque si bien el currículo preescrito, a través de los programas de estudio, propicia y propone al profesorado la enseñanza de ciertos conocimientos, habilidades y actitudes ciudadanas, en la práctica el personal docente no los incorpora en el aula, con una mirada ciudadana. Lo anterior hace que la escuela aparezca ensimismada en una lógica más bien disciplinar, en donde los contenidos tienen validez en tanto son prescritos curricularmente para ser enseñados en forma individual, pero no desde su valor de uso en el contexto social, al que los propios profesores y estudiantes pertenecen y en el que la escuela está inmersa. Esto trae, como consecuencia, que los estudiantes yuxtapongan sus legítimos intereses con los de la sociedad, contradiciendo así los objetivos que persigue la formación ciudadana en el sistema escolar, que busca que la escuela sea un espacio donde los estudiantes aprendan a vivir en sociedad, más allá de los contenidos preescritos en el currículo de formación.

La tarea es compleja, porque no solo se trata de efectuar una adecuada cobertura curricular de los contenidos vinculados a la temática ciudadana, sino que también de ofrecer al estudiantado la oportunidad de vivir en la escuela los principios y valores vinculados a ella. Asumir este desafío implica revisar y enfrentar de manera distinta la transposición didáctica de todos los contenidos de enseñanza y no solo de aquellos vinculados directamente a la formación ciudadana. También implica hacer del aula un espacio de trabajo donde el respeto, la tolerancia, la participación, la colaboración, la inclusión y la posibilidad de tomar decisiones conjuntas e informadas constituyan experiencias y vivencias cotidianas por parte del estudiantado y no solo conceptos a ser aprendidos en forma memorística. De tal manera que asumir este desafío implica una revisión de los planeamientos didácticos y de la transposición que se encuentra a la base de ellos, también de las oportunidades de aprendizaje realmente ofrecidas, del rol y el nivel de participación estudiantil y del profesorado, del uso de fuentes variadas y de la posibilidad de llevar al aula un conocimiento inacabado y en permanente revisión.

Los resultados obtenidos también sugieren que, a pesar de los esfuerzos ministeriales realizados durante las últimas décadas, los grupos de estudiantes siguen vinculando la formación ciudadana casi exclusivamente al ámbito político, lo que si bien es coherente con el enfoque minimalista descrito en la bibliografía especializada, se contrapone al enfoque maximalista que se promueve desde el currículo escolar, retrotrayendo la situación al currículo promovido durante la dictadura.

Vincular la formación ciudadana de manera exclusiva a lo político es promover la formación de una ciudadanía que no es capaz de vivir ni reconocer la importancia de su presencia y actuación en otros ámbitos tan importantes como el social, el económico, el cultural y el espiritual. También es pensar en lo político como la única y casi exclusiva forma de participación en una sociedad, es considerar al ciudadano como un elector más bien pasivo que concurre cada cierto tiempo a sufragar, es promover una democracia representativa más que participativa que superpone la gobernabilidad a la gobernanza, es valorar el capital electoral por sobre el capital social; en fin, es pensar que la vida de un ciudadano o ciudadana se inicia 
doi: http://dx.doi.org/10.15359/ree.18-2.12

URL: http://www.una.ac.cr/educare

CORREO: educare@una.cr

y se acaba en lo político, en lo electoral y en lo legal, en consecuencia que somos parte de aquellos que piensan que un buen ciudadano es quien es capaz de vivir con las otras personas y junto a ellas, en un espacio de construcción colectiva y cooperativa que llamamos sociedad.

\section{Referencias}

Andrade, M. y Miranda, J. (2000). El concepto de ciudadanía en educación. Análisis semiótico de las representaciones sociales del concepto de ciudadanía en profesores mapuches y citadinos de educación general básica en Chile. Trabajo presentado en el 13a Congreso Mundial de Educación (AMSE), Université de Sherbrooke, Québec, Canadá. Recuperado de http://200.10.23.169/documentos/documentos\%20originales/nuevos sentidos concepto ciudadania educacion.pdf

Apple, M. (2004). Ideology and curriculum [Ideología y currículo]. New York: Falmer.

Bardin, L. (1996). Análisis de contenido (2a ed.). Madrid: Ediciones Akal.

Bauman, Z. (enero-marzo, 2005). La ambivalencia revisitada. Algunas palabras para los lectores de lengua española. Revista Anthropos: Huellas del conocimiento, 206, 31-35.

Beck, U. (1993). La invención de lo político. Para una teoría de la modernización reflexiva. Buenos Aires: Fondo de Cultura Económica.

Bolívar, A. (2007). Educación para la ciudadanía. Algo más que una asignatura. Barcelona: Graó.

Cerda, A. M., Loreto, M., Magendzo, A., Santa, E. y Varas, R. (2004). El complejo camino de la formación ciudadana. Una mirada a las prácticas docentes. Santiago: Lom ediciones.

Cortina, A. (1997). Ciudadanos del mundo: Hacia una teoría de la ciudadanía. Madrid: Alianza.

Cox, C., Jaramillo, R. y Reimers, F. (2005). Educar para la ciudadanía y la democracia en las Américas: Una agenda para la acción. Washington: Banco Interamericano de Desarrollo. Recuperado de http://www.educadem.oas.org/documentos/getdocument.pdf

Díaz-Barriga, F. y Hernández, G. (2005). Estrategias docentes para un aprendizaje significativo. México: MacGraw-Hill.

Egaña L. (2003). Reforma educativa y objetivos fundamentales transversales. Los dilemas de la innovación. Santiago: PIIE.

Kerr, D. (2002). An International Review of Citizenship in the Curriculum: The IEA National Case Studies and the INCA Archive [Una Revista Internacional de Ciudadanía en el Plan de Estudios: Los estudios de caso de la AIE Nacionales y el Archivo INCA]. En G. SteinerKhamsi, J. V. Torney-Purta y J. Schwille (Eds.), New Paradigms and Recurring Paradoxes in Education for Citizenship: An International Comparison [Nuevos Paradigmas y Paradojas recurrentes en educación para la ciudadanía: Una comparación internacional] (pp. 207-237). Amsterdam: Elsevier Pres. 
doi: http://dx.doi.org/10.15359/ree.18-2.12

URL: http://www.una.ac.cr/educare

CORREO: educare@una.cr

Lundgren, U. P. (1997). Teoría del curriculum y escolarización. Madrid: Morata.

Magendzo A. (2004). Educación y ciudadanía: Construyendo ciudadanía desde el currículum oficial y la cotidianidad de la escuela. Una lectura desde la investigación. Santiago: PIIE.

MINEDUC. (2004a). Educación cívica y el ejercicio de la ciudadanía. Los estudiantes chilenos en el estudio internacional de educación cívica. Santiago: Unidad de Currículum y Evaluación. Recuperado de http://www.oei.es/quipu/chile/eval civica.pdf

MINEDUC (2004b). Formación ciudadana en el currículo de la reforma. Santiago: Unidad de Currículum y Evaluación. Recuperado de http://es.scribd.com/doc/37270503/TercerLibro-Formacion-Ciudadana-en-el-Curriculum-de-la-Reforma

MINEDUC (2004c). Informe comisión formación ciudadana. Santiago: Autor.

Muñoz, C., Ajagan L., Saéz, G., Cea R., Luengo H. (2013a). Relaciones dialécticas antagónicas entre la cultura escolar y la cultura familiar de niños y niñas de 1(28), 129-148. Recuperado de http://universum.utalca.cl/contenido/index-13-1/Articulo 5 Munoz et al.html

Muñoz, C., Vásquez, N. y Reyes, L. (2010a). Percepción del estudiantado de enseñanza básica sobre el rol del estado, las instituciones públicas, la democracia, la ciudadanía y los derechos de las mujeres y de los inmigrantes. Estudios Pedagógicos, 36(2), 153-175. Recuperado de http://mingaonline.uach.cl/pdf/estped/v36n2/art09.pdf

Muñoz C., Vásquez, N. y Sánchez, M. (2013b). Percepciones del estudiantado sobre la democracia y los derechos humanos al finalizar la educación general básica. Un estudio desde las aulas de historia. Psicoperspectivas, 12(1), 95-115. Recuperado de http://www.psicoperspectivas. cl/index.php/psicoperspectivas/article/viewFile/230/248

Muñoz C., Victoriano R., Sánchez M., Wilhelm R. (2010b). El estudiantado y la formación ciudadana en la escuela. Recuperado de http://es.scribd.com/doc/37268630/FONIDE-Elestudiantado-y-la-Formacion-Ciudadana

Stake R. (2010). Qualitative Research. Studying How Things Work [Investigación cualitativa. Estudiando cómo funcionan las cosas]. New York: Guilford Press.

(6) Cómo citar este artículo en APA:

Muñoz, C. y Torres, B. (mayo-agosto, 2014). La formación ciudadana en la escuela: Problemas y desafíos. Revista Electrónica Educare, 18(2), 233-245. doi: http://dx.doi.org/10.15359/ree.18-2.12

Nota: Para citar este artículo en otros sistemas puede consultar el hipervínculo "Como citar el artículo" en la barra derecha de nuestro sitio web:

http://www.revistas.una.ac.cr/index.php/EDUCARE/index 